\title{
Analytical Study of Creeping Flow past a Composite Sphere: Solid Core with Porous Shell in Presence of Magnetic Field
}

\author{
D.V. Jayalakshmamma, ${ }^{*}$ P.A. Dinesh $\dagger$ and M. Sankarł
}

\begin{abstract}
The two-dimensional creeping flow of an electrically conducting fluid past a multiple composite sphere consists of solid sphere with permeable porous shell in presence of applied transverse magnetic field is studied analytically using Stokes and Brinkman equations. The basic governing equations are solved using similarity solution method and closed forms of exact solutions are obtained. Stream lines are discussed for various Hartmann number $(M)$ and porous parameter $(\sigma)$. It is observed that the fluid flow is suppressed in presence of magnetic field both in non-porous and porous regions and also the results obtained are in excellent agreement in the absence of the magnetic field.
\end{abstract}

Keywords: Stokes equation, Brinkman equation, creeping flow, magnetic field.

\section{Introduction}

The problem of flow through / past porous media have several important applications, like the flow of oil through rocks,

\footnotetext{
* Vemana Institute of Technology, Bangalore 560 034; jaya.dvj@gmail.com

†M.S. Ramaiah Institute of Technology, Bangalore 560 054; dineshdpa@hotmail.com

‡ East Point College of Engineering and Technology, Bangalore 560 049; manisankarir@yahoo.com
} 
extraction of energy from geothermal region, combustion in an inert porous matrix, underground spreading of chemical waste, chemical catalytic reactors, the filtration of solids from liquids, the study of flow sedimentation. Also, the drug permeation through human skin, dispersion of cholesterol and other fat substances from arteries to endothelium. To understand the concept of these practical application one should know fluid flow past / through a body, in particular fluid flow past / through a sphere or cylinder in porous region.

For the above cited applications, we must apply a constitutive equation to describe the fluid velocity and hydrodynamic pressure for the considered domain. For creeping flow (low Reynolds number), there are two methods commonly employed namely Darcy's law and the Brinkman's equation. In the literature, we find several studies on the flow past a sphere in porous media using Darcy's or Brinkman equation under different boundary conditions. Beaver's and Joseph (1967), Saffman (1971), Raja Sekhar and Amarnath (1996), where they have used Darcy's equation to describe the flow field. The problem of stokes flow past porous bodies have been studied by Higdon and Kojima (1981), Qin and Kaloni (1988), Pop and Cheng (1992), Padmavathi et al. (1993) Barman (1996), Pop and Ingham (1996), using Brinkman equation in the porous region.

Many works have been done on the flow past / through a composite sphere using Stoke's and Brinkman equations under different boundary conditions. Masliyah (1987) conducted a theoretical analysis to the creeping flow over an isolated composite sphere with a permeable shell, whose permeability is constant, but thickness can arbitrary. The drag experienced by a composite sphere is derived using the Brinkman flow equation together with Stoke's flow approximation. Kaloni and Qin (1993) have used continuity of velocity, stress and continuity of pressure has been used instead of continuity of normal stress in discussing uniform flow past a spherical shell. The axisymmetric motion of multiple composite spheres, solid core with permeable shell under creeping flow condition was studied by Shing Bor Chen (1998) to investigate the hydrodynamic interactions for a chain of composite spheres undergoing axisymmetric motion. The Stokes and Brinkman 
equations are used to describe the flow field outside and inside particle respectively. An arbitrary flow of viscous, incompressible fluid past a porous sphere of radius $a$ with an impermeable core of radius $b$, is studied using Brinkman equation in the porous region by Anindita Bhattacharyya and Raja Sekhar (2004) with stress jump condition at the interface of the fluid-porous region. They found that the drag and torque not only change with the change of permeability but also significant effect of stress jump coefficient is observed. Srivastava and Srivastava (2005) have discussed the steady flow of an incompressible viscous fluid streaming past a sphere at small Reynolds number with a uniform velocity by dividing the flow in three regions and in all the three regions Stoke's stream function is expressed in powers of Reynolds number. Recently Pop, et.al (2010) studied Brinkman flow of a viscous fluid through spherical porous medium embedded in another porous medium using the continuity of velocity, stress and normal stress at the interface. They have shown that the dimensionless shearing stress on the sphere is periodic in nature and its absolute value increases with an increase of porous parameters in both the regions.

Many practical problems need a mechanism to control motion of the fluid past solid bodies with MHD effects. Stewartson (1956), Childress (1963), Sanyal and Roy Chowdhury (1984), have studied the flow of an electrically conducting incompressible fluid past a sphere / cylinder in presence of a magnetic field.

In the available literature less attention has been reported on the flow past a composite sphere in presence of external constraints, which accelerates or suppresses the flow past / through a body. In this article we studied the creeping flow of a steady, incompressible, viscous, electrically conducting fluid past a solid sphere embedded in porous medium in presence of transverse magnetic field with the boundary conditions as continuity of velocity, tangential and normal stress at the interface of the nonporous and porous region and no-slip condition at the surface of the solid sphere. We observed that the presence of the magnetic field suppress the flow in the porous region as well as in the nonporous region. 


\section{Mathematical formulation}

Consider a steady, incompressible, electrically conducting, viscous fluid past a stationary solid sphere of radius ' $a$ ' embedded in a porous sphere of radius $b$ in presence of uniform magnetic field $\left(H_{0}\right)$ in transverse direction as shown in the physical configuration fig.1. Here we use the spherical co-ordinate system $(r, \theta, \emptyset)$ with the origin at the center of the sphere and the axis $\theta=0$ along the direction of the uniform flow $u_{\infty}$ from the porous sphere and due to axisymmetry we have $\frac{\partial}{\partial \emptyset}=0$. Also we consider the flow domain in two regions as non-porous and porous region.

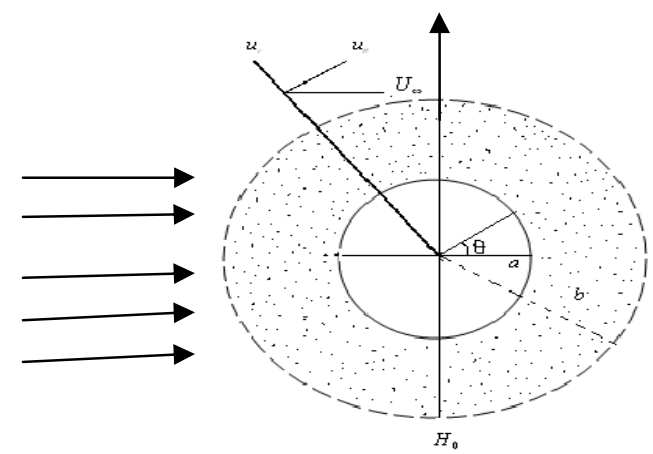

Figure1: Physical configuration

The constitutive equations for the flow in non-porous region is given by

$$
\begin{gathered}
\nabla \cdot \vec{q}_{1}=0, \\
\mu_{h}^{2} \sigma_{e}\left(\vec{q}_{1} \times \vec{H}\right) \times \vec{H}+\mu \nabla^{2} \vec{q}_{1}=\nabla p_{1},
\end{gathered}
$$

where $\vec{q}_{1}=\left(u_{r}, u_{\theta}, u_{\emptyset}\right)$ is the velocity of the non-porous region, $\mu$ is the viscosity of the fluid, $\mu_{h}$ is the magnetic permeability, $\sigma_{e}$ is electrical conductivity, $\vec{H}$ transverse magnetic field and $p_{1}$ is hydrostatic pressure of the non-porous region.

For the flow in the porous region is described by

$$
\nabla \cdot \vec{q}_{2}=0,
$$




$$
-\frac{\mu}{k} \vec{q}_{2}+\mu_{h}^{2} \sigma_{e}\left(\vec{q}_{2} \times \vec{H}\right) \times \vec{H}+\mu^{*} \nabla^{2} \vec{q}_{2}=\nabla p_{2}
$$

where $\vec{q}_{2}=\left(v_{r}, v_{\theta}, v_{\emptyset}\right)$ is the velocity of the fluid in the porous region, $\mu^{*}$ is the Brinkman viscosity, $p_{2}$ is hydrostatic pressure of the fluid in the porous region and $k$ is the permeability of the porous region. Here we assume that Brinkman viscosity is equal to viscosity of the fluid i,e $\mu^{*}=\mu$.

Making the equations (1) to (4) dimensionless using the dimensionless parameters as

$$
r^{*}=\frac{r}{a}, \quad \vec{q}_{1}^{*}=\frac{\vec{q}_{1}}{u_{\infty}}, \quad \vec{q}_{2}^{*}=\frac{\vec{q}_{2}}{u_{\infty}}, \quad p_{1}^{*}=\frac{a p_{1}}{\mu u_{\infty}}, \quad p_{2}^{*}=\frac{a p_{2}}{\mu^{*} u_{\infty}}, \quad \vec{H}^{*}=\frac{\vec{H}}{H_{0}},
$$

Using the dimensionless variables from equation (5) in equations (1) and (2), we get

$$
\begin{gathered}
\frac{\partial}{\partial r}\left(r^{2} u_{r}\right)+\frac{r}{\sin \theta} \frac{\partial}{\partial \theta}\left(u_{\theta} \sin \theta\right)=0, \\
\frac{\partial p_{1}}{\partial r}=M^{2} u_{r}+\left[\frac{\partial^{2} u_{r}}{\partial r^{2}}+\frac{2}{r} \frac{\partial u_{r}}{\partial r}+\frac{1}{r^{2}} \frac{\partial^{2} u_{r}}{\partial \theta^{2}}+\frac{\cot \theta}{r^{2}} \frac{\partial u_{r}}{\partial \theta}-\frac{2 u_{r}}{r^{2}}-\frac{2}{r^{2}} \frac{\partial u_{\theta}}{\partial \theta}-\frac{2 u_{\theta} \cot \theta}{r^{2}}\right] \\
\frac{1}{r} \frac{\partial p_{1}}{\partial \theta}=M^{2} u_{\theta}+\left[\frac{\partial^{2} u_{\theta}}{\partial r^{2}}+\frac{2}{r} \frac{\partial u_{\theta}}{\partial r}+\frac{1}{r^{2}} \frac{\partial^{2} u_{\theta}}{\partial \theta^{2}}+\frac{\cot \theta}{r^{2}} \frac{\partial u_{\theta}}{\partial \theta}+\frac{2}{r^{2}} \frac{\partial u_{r}}{\partial \theta}-\frac{u_{\theta} \operatorname{cosec}^{2} \theta}{r^{2}}\right]
\end{gathered}
$$

nwhere $M^{2}=\frac{\mu_{h}^{2} \sigma_{e} H_{0}^{2} a^{2}}{\mu}$ is the Hartmann number.

Similarly non-dimensionalising equations (3) and (4) using equation (5), we get

$$
\begin{gathered}
\frac{\partial}{\partial r}\left(r^{2} v_{r}\right)+\frac{r}{\sin \theta} \frac{\partial}{\partial \theta}\left(v_{\theta} \sin \theta\right)=0 \\
-\frac{\partial p_{1}}{\partial r}=S^{2} v_{r}-\left[\frac{\partial^{2} v_{r}}{\partial r^{2}}+\frac{2}{r} \frac{\partial v_{r}}{\partial r}+\frac{1}{r^{2}} \frac{\partial^{2} v_{r}}{\partial \theta^{2}}+\frac{\cot \theta}{r^{2}} \frac{\partial v_{r}}{\partial \theta}-\frac{2 v_{r}}{r^{2}}-\frac{2}{r^{2}} \frac{\partial v_{\theta}}{\partial \theta}-\frac{2 v_{\theta} \cot \theta}{r^{2}}\right] \\
-\frac{1}{r} \frac{\partial p_{1}}{\partial \theta}=S^{2} v_{\theta}-\left[\frac{\partial^{2} v_{\theta}}{\partial r^{2}}+\frac{2}{r} \frac{\partial v_{\theta}}{\partial r}+\frac{1}{r^{2}} \frac{\partial^{2} v_{\theta}}{\partial \theta^{2}}+\frac{\cot \theta}{r^{2}} \frac{\partial v_{\theta}}{\partial \theta}+\frac{2}{r^{2}} \frac{\partial v_{r}}{\partial \theta}-\frac{v_{\theta} \operatorname{cosec}^{2} \theta}{r^{2}}\right]
\end{gathered}
$$

where $S^{2}=\sigma^{2}+M^{2}, \sigma=\frac{a}{\sqrt{k}}$ is the porous parameter.

By introducing the stream function $\Psi_{1}(r, \theta)$ in the non-porous region, which satisfies equation of continuity, equation (6) as

$$
u_{r}=\frac{1}{r^{2} \sin \theta} \frac{\partial \Psi_{1}}{\partial \theta} ; \quad u_{\theta}=\frac{-1}{r \sin \theta} \frac{\partial \Psi_{1}}{\partial r} .
$$


By eliminating the pressure from equation (7) and (8) using equation (12), we get

$$
E^{4} \Psi_{1}-M^{2} E^{2} \Psi_{1}=0, \quad b \leq r<\infty .
$$

Similarly in porous region $\Psi_{2}(r, \theta)$ represent the stream function which satisfies equation of continuity in porous region, equation (9) as

$$
v_{r}=\frac{1}{r^{2} \sin \theta} \frac{\partial \Psi_{2}}{\partial \theta} ; \quad v_{\theta}=\frac{-1}{r \sin \theta} \frac{\partial \Psi_{2}}{\partial r} .
$$

By eliminating the pressure from equation (10) and (11) using (14) we get

$$
E^{4} \Psi_{2}-S^{2} E^{2} \Psi_{2}=0, \quad a \leq r \leq b .
$$

where $E^{2}=\frac{\partial^{2}}{\partial r^{2}}+\frac{\sin \theta}{r^{2}} \frac{\partial}{\partial \theta}\left(\frac{1}{\sin \theta} \frac{\partial}{\partial \theta}\right)$ is an operator.

To solve equation (13) and (15), we considered the following boundary conditions, the no-slip condition at solid sphere

$$
\begin{array}{ll}
v_{r}(a, \theta)=0, & 0 \leq \theta \leq 2 \pi, \\
v_{\theta}(a, \theta)=0, & 0 \leq \theta \leq 2 \pi,
\end{array}
$$

the matching condition at the interface of the non-porous and porous region

$$
\begin{aligned}
& v_{r}(b, \theta)=u_{r}(b, \theta), \quad 0 \leq \theta \leq 2 \pi, \\
& v_{\theta}(b, \theta)=u_{\theta}(b, \theta), \quad 0 \leq \theta \leq 2 \pi, \\
& \tau_{r \theta(2)}(b, \theta)=\tau_{r \theta(1)}(b, \theta), \quad 0 \leq \theta \leq 2 \pi, \\
& \tau_{r r(2)}(b, \theta)=\tau_{r r(1)}(b, \theta), \quad 0 \leq \theta \leq 2 \pi .
\end{aligned}
$$

We know that the stream function in the non-porous region, is

$$
\Psi_{1}(r, \theta)=\frac{1}{2}\left(r^{2}-\frac{1}{r}\right) \sin ^{2} \theta, \quad b \leq r<\infty .
$$

Using equations (12) and (22), the boundary conditions for the velocity components in the non-porous region are

$$
u_{r} \sim \cos \theta \text { and } u_{\theta} \sim-\sin \theta \text { as } r \rightarrow \infty .
$$


Hence the boundary condition far from the porous region equation (22) in terms of $\Psi_{1}$ is

$$
\Psi_{1}(r, \theta) \sim \frac{r^{2}}{2} \sin ^{2} \theta \quad \text { as } \quad r \rightarrow \infty .
$$

\section{Method of Solution}

The boundary condition equation(24) suggests the following similarity solution to equation (13) and (15) as

$$
\begin{array}{ll}
\Psi_{1}(r, \theta)=f_{1}(r) \sin ^{2} \theta, & b \leq r<\infty, \\
\Psi_{2}(r, \theta)=f_{2}(r) \sin ^{2} \theta, & a \leq r \leq b .
\end{array}
$$

Substituting equation (25) in (13) and equation (26) in (15), the partial differential equation of order four in terms of respective stream functions $\Psi_{1}(r, \theta)$ and $\Psi_{2}(r, \theta)$ reduces to ordinary differential equation of order four in $f_{1}(r)$ and $f_{2}(r)$ respectively

$$
\begin{aligned}
& f_{1}^{i v}(r)-\frac{4 f_{1}^{\prime \prime}(r)}{r^{2}}+\frac{8 f_{1}^{\prime}(r)}{r^{3}}-\frac{8 f_{1}(r)}{r^{4}}-M^{2}\left(f_{1}^{\prime \prime}(r)-\frac{2 f_{1}(r)}{r^{2}}\right)=0, b \leq r<\infty, \\
& f_{2}^{i v}(r)-\frac{4 f_{2}^{\prime \prime}(r)}{r^{2}}+\frac{8 f_{2}^{\prime}(r)}{r^{3}}-\frac{8 f_{2}(r)}{r^{4}}-S^{2}\left(f_{2}^{\prime \prime}(r)-\frac{2 f_{2}(r)}{r^{2}}\right)=0 \quad a \leq r \leq b .
\end{aligned}
$$

And also, the boundary conditions in terms of stream functions reduces in $f_{1}(r)$ and $f_{2}(r)$ as no-slip condition at the surface of the solid sphere

$$
\begin{aligned}
& f_{2}(a)=0, \\
& f_{2}^{\prime}(a)=0,
\end{aligned}
$$

the matching condition at the interface of the porous and nonporous region

$$
\begin{gathered}
f_{2}(b)=f_{1}(b), \\
f_{2}^{\prime}(b)=f_{1}^{\prime}(b), \\
f_{2}^{\prime \prime}(b)=f_{1}^{\prime \prime}(b), \\
f_{2}^{\prime \prime \prime}(b)-\sigma^{2} f_{2}^{\prime}(b)=f_{1}^{\prime \prime \prime}(b),
\end{gathered}
$$


and for far from the boundary we get from equation (24) as

$$
f_{1}(r) \sim \frac{r^{2}}{2} \quad \text { as } \quad r \rightarrow \infty
$$

Solving equations (27) and (28), we obtained the solution in the form

$$
\begin{array}{r}
f_{1}(r)=\frac{A}{r}+B r^{2}+C \sqrt{\frac{\pi}{2}}\left(1+\frac{1}{M r}\right) e^{-M r}, \\
f_{2}(r)=\frac{D}{r}+E r^{2}+F\left(\frac{\cosh S r}{S r}-\sinh S r\right)+G\left(\frac{\sinh S r}{S r}-\cosh S r\right)
\end{array}
$$

where $A, B, C, D, E, F, G$ are arbitrary constants to be determined using the boundary conditions from equations (29) to (35) and the obtained results are given in the appendix.

\section{Results and discussions}

We considered the study of steady flow of an incompressible, viscous, electrically conducting fluid past a solid sphere embedded in a porous medium in presence of uniform magnetic field in the transverse direction of the fluid motion. The stream functions are obtained for non-porous and porous region.

The nature of stream lines are observed by giving different Hartmann numbers for a small fixed porous parameter to understand the effect of the magnetic field and also we observed the flow pattern for negligible Hartmann number by varying porous parameter. From fig. 2(a) and 2(b) we notice that for a small porous parameter $\sigma=2$ and for Hartmann number $M=1,2$, the flow of the fluid through porous medium is more and moves over the surface of the solid sphere. As the Hartmann number increases for $M=10$, the flow of the fluid in the porous region is reduced and moves away from the solid sphere which is shown in fig. 2(c). And further increase in Hartmann number for $M=50$, fig. 2(d) shows the flow of the fluid past a porous sphere. Therefore, in presence of magnetic field the fluid flow is supressed in both the regions. 

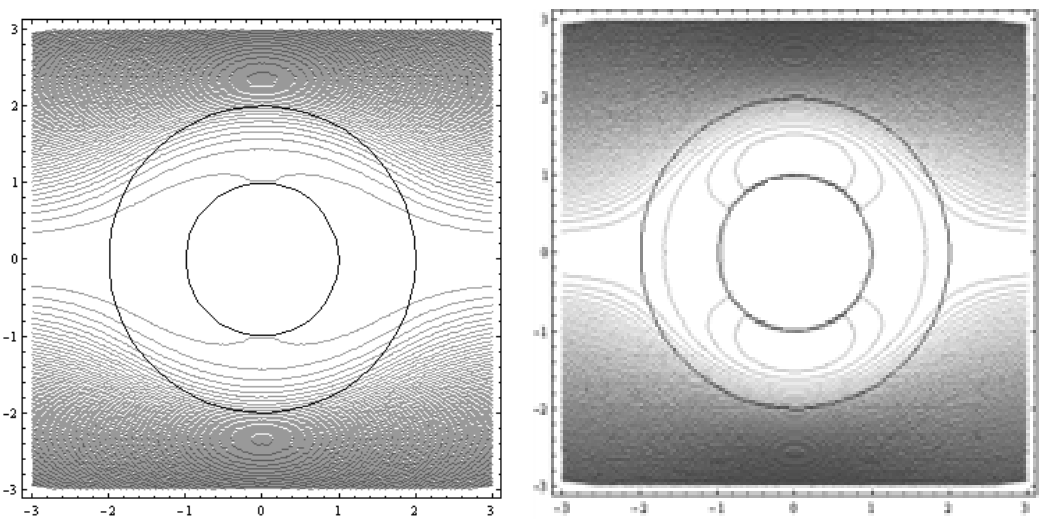

(a) $M=1$ and $\sigma=2$

(b) $M=2$ and $\sigma=2$
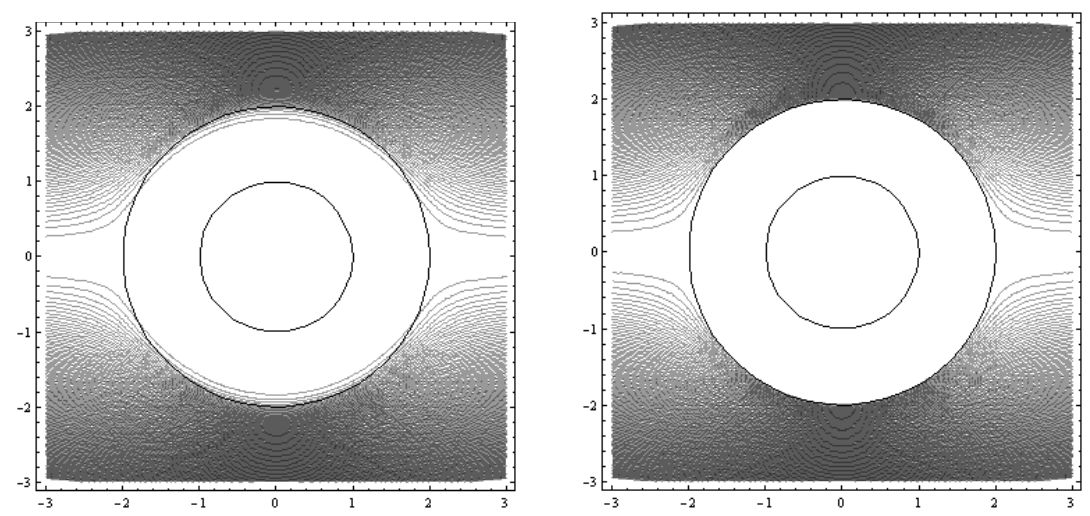

(c) $M=10$ and $\sigma=2$

(d) $M=50$ and $\sigma=2$

Figure 2 (a)-(d): Stream lines for variation of Hartmann number $M=$ $1,2,10$ and 50 for fixed porous parameter $\sigma=2$.

To know the effect of fluid flow in absence of magnetic field, we have chosen negligibly small Hartmann number (approximately equal to zero) and vary the porous parameter. It is evident from the fig.3(a)-(d) where the flow of the fluid is supressed in porous and non-porous region due to the effect of porous parameter, which was observed fluid flow in the earlier works. 


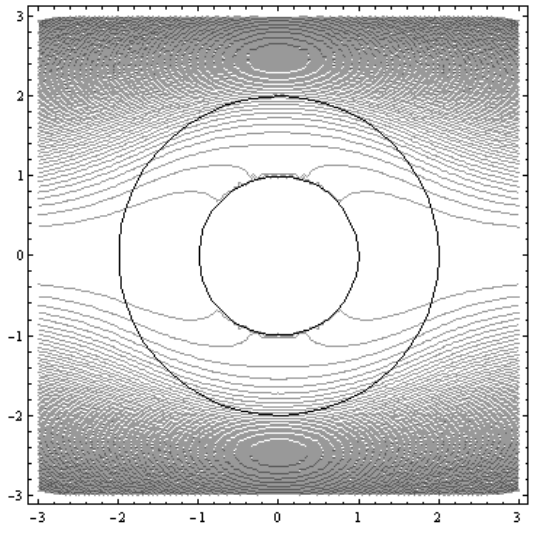

(a) $M=0.001$ and $\sigma=2$

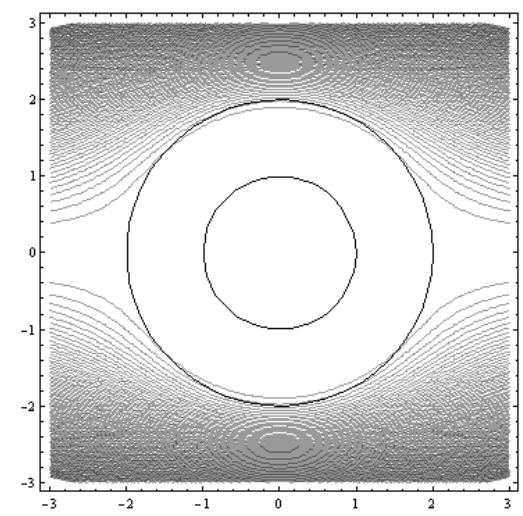

(c) $M=0.001$ and $\sigma=10$

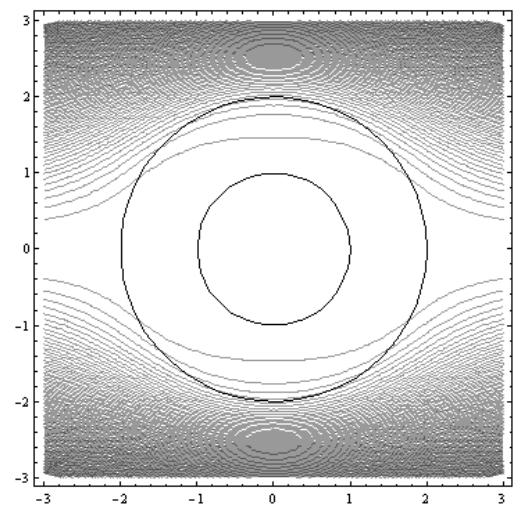

(b) $\quad M=0.001$ and $\sigma=5$

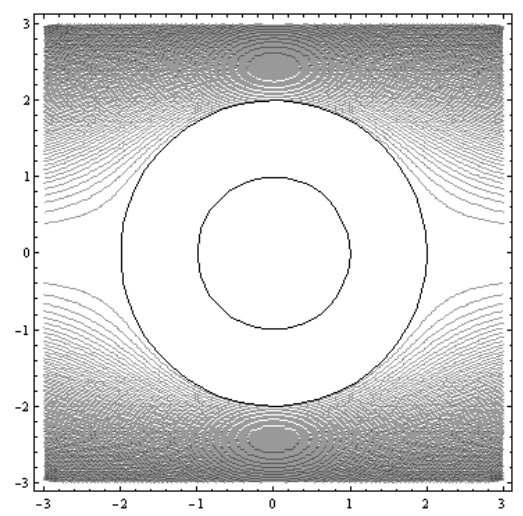

(d) $M=0.001$ and $\sigma=50$

Figure 3 (a)-(d): Stream lines for variation of porous parameter $\sigma=2,5,10$ and 50 for neligible Hartmann number $M=0.001$.

\section{Conclusion}

In this article, the stream lines are plotted by varying the Hartmann number keeping the porous parameter constant, and we found that the fluid flow is supressed in non-porous the porous regions in presence of the magnetic field. Also the results are in excellent agreement with the existing literature in absence of magnetic field (for negligibly small Hartmann number). 


\section{Acknowledgement}

The authors are grateful to the research center M S Ramaiah Institute of Technology, Vemana Institute of Technology and East Point College of Engineering and Technology for their support and encouragement and also the financial support from VTU research scheme.

\section{References}

[1] A Bhattacharyya and Raja Sekhar G.P., (2004): "Viscous flow past a porous sphere with an impermeable core: effect of stress jump condition", Chemical Engineering Science, Vol. 59, pp. 4481-4492

[2] Bhupen Barman., (1996): "Flow of a Newtonian fluid past an impervious sphere embedded in porous medium", Indian J. pure Appl.Math, Vol.27(12), pp. 1249-1256.

[3] Breavers, G. S., and Joseph, D.D., (1967): "Boundary conditions at a naturally permeable wall", J. Fluid Mech, Vol.30(1), pp.197-207.

[4] Childress S., (1972): "Viscous flow past an array of spheres", J. Chem. Phys. Vol. 56, pp 2527-2539.

[5] Grosan T. Postelnicu A and Pop I., (2010): “Brinkman flow of a viscous Fluid through a spherical porous medium embedded in another porous medium", Transp. Porous Med. Vol. 81, pp. 89-103.

[6] Higdon, J.J.L., Kojima, M., (1981): “On the calculation of stokes flow past porous particles", Int. J. Multiphase flow, vol.7 (6), pp. 719-727.

[7] Masliyah, Neale J H, Malysa G M, and Van De Ven YGM., (1987): "Creeping flow over a composite sphere: Solid core with porous shell", Chem. Engg. Sci, Vol. 42(2) pp. 245-253

[8] Padmavathi B S, Amaranath T and Nigam S.D., (1993): "Stokes flow past a porous sphere using Brinkman model", ZAMP, Vol. 44, pp. 929939.

[9] Pop I., Cheng P., (1992): “Flow past a circular cylinder embedded in a porous medium based on the Brinkman model", Int. J. Engg. Sci, Vol. 30(2), pp. 257-262.

[10] Pop I., Ingham D.B., (1996): "Flow past a sphere embedded in a porous medium based on Brinkman model", Int. Comm. Heat Mass Trans, Vol. 23 (6), pp. 865-874. 
[11] Qin Yu and Kaloni P. N., (1988): "A Cartesian tensor solution of the Brinkman equation", J. Engg. Math, Vol. 22 pp. 177-188.

[12] Qin Yu and Kaloni P. N., (1993); "Creeping flow past a porous spherical shell", ZAMM, Vol. 73, pp. 77-84.

[13] Raja Sekhar G.P., and Amaranath T., (1996): "Stokes flow past a porous sphere with an impermeable core", Mechanics Research Communications, Vol. 23(5), pp. 449-460.

[14] Roy Chowdhury S.K and Sanyal D.C., (1984): "Magneto- Shear flow past a cylinder of section bounded by two circular arcs", J. Indian Inst. Sc. Vol. 65(B) pp. 9-19.

[15] Saffman P. G., (1971): "On the boundary condition at the surface of a porous medium", Studies in Appl. Math., Vol. 50, pp. 93-101.

[16] Shing Bor Chen, (1998): "Axisymmetric motion of multiple composite spheres: solid core with permeable shell, under creeping flow condition", Physics of fluids. Vol. 10(7) pp. 1550-1563

[17] Srivastava, A. C and Srivastava N., (2005): "Flow past a porous sphere at small Reynolds number", ZAMP, Vol. 56, pp. 821-835

[18] Stewartson K., (1956): "Motion of a sphere through a conducting fluid in the presence of strong magnetic field", J. Fluid Mech., pp. 301-316.

\section{Appendix}

$$
\begin{aligned}
& G=\frac{m_{1} m_{6}-m_{4} m_{3}}{m_{1} m_{5}-m_{4} m_{2}} ; F=\frac{1}{m_{1}}\left(m_{3}-m_{2} G\right) ; \\
& E=\frac{1}{n_{1}}\left(n_{4}-n_{3} G-n_{2} F\right) ; \\
& D=\frac{1}{n_{5}}\left(n_{9}-n_{8} G-n_{7} F-n_{6} E\right) ; \\
& C=\frac{1}{l_{1}-l_{5}}\left[\frac{-3 l_{2}}{2}+\left(l_{4}+l_{7}\right) G+\left(l_{3}+l_{6}\right) F+3 l_{2} E\right] ; \\
& B=\frac{1}{2} ; \\
& A=\frac{-l_{2}}{2}+l_{4} G+l_{3} F+l_{2} E+D-l_{1} C ; \text { where } \\
& m_{1}=n_{1}\left(n_{5} l_{18}-n_{7}\right)-n_{1}\left(n_{5} l_{20}-n_{6}\right) ;
\end{aligned}
$$




$$
\begin{aligned}
& m_{2}=n_{1}\left(n_{5} l_{17}-n_{8}\right)-n_{3}\left(n_{5} l_{20}-n_{6}\right) ; \\
& m_{3}=-n_{1} n_{9}-n_{4}\left(n_{5} l_{20}-n_{6}\right) ; \\
& m_{4}=n_{1}\left(n_{5} l_{18}+n_{7}\right)-n_{2}\left(2 n_{5} l_{20}+n_{6}\right) ; \\
& m_{5}=n_{1}\left(n_{5} l_{19}+n_{8}\right)-n_{3}\left(2 n_{5} l_{20}+n_{6}\right) ; \\
& m_{6}=n_{1} n_{9}-n_{4}\left(2 n_{5} l_{20}+n_{6}\right) ; \\
& n_{1}=3 l_{2}\left(l_{8}-2 l_{1}\right) ; n_{2}=\left(2 l_{2}-l_{9}\right)\left(l_{1}-l_{5}\right)+\left(l_{8}-2 l_{1}\right)\left(l_{3}+l_{6}\right) ; \\
& n_{3}=\left(2 l_{4}-l_{10}\right)\left(l_{1}-l_{5}\right)+\left(l_{4}+l_{7}\right)\left(l_{8}-2 l_{1}\right) ; \\
& n_{4}=\frac{3 l_{2}}{2}\left(l_{8}-2 l_{1}\right) ; n_{5}=\left(l_{12}-6\right)\left(l_{1}-l_{5}\right) ; \\
& n_{6}=\left(l_{13}-6 l_{2}\right)\left(l_{1}-l_{5}\right)+3 l_{2}\left(6 l_{1}-l_{11}\right) ; \\
& n_{7}=\left(l_{14}-6 l_{3}\right)\left(l_{1}-l_{5}\right)+\left(l_{3}+l_{6}\right)\left(6 l_{1}-l_{11}\right) ; \\
& n_{8}=\left(l_{15}-6 l_{4}\right)\left(l_{1}-l_{5}\right)+\left(l_{4}+l_{7}\right)\left(6 l_{1}-l_{11}\right) ; \\
& n_{9}=-3 l_{2}\left(l_{1}-l_{5}\right)+\frac{3 l_{2}}{2}\left(6 l_{1}-l_{11}\right) ; \\
& l_{1}=\sqrt{\frac{\pi}{2}}\left(b+\frac{1}{M}\right) e^{-M b} ; \\
& l_{2}=b^{3} ; \\
& l_{3}=\frac{\cosh S b}{s b}-b \sinh S b ; \\
& l_{4}=\frac{\sinh S b}{s b}-b \cosh S b ; \\
& l_{5}=\sqrt{\frac{\pi}{2}} M\left(b^{2}+\frac{b}{M}+\frac{1}{M^{2}}\right) e^{-M b} ; \\
& l_{6}=b \sinh S b-\left(\frac{1}{s}+S b^{2}\right) \cosh S b ; \\
& l_{7}=b \cosh S b-\left(\frac{1}{S}+S b^{2}\right) \sinh S b ;
\end{aligned}
$$




$$
\begin{aligned}
& l_{8}=\sqrt{\frac{\pi}{2}} M^{2}\left(b^{3}+\frac{b^{2}}{M}+\frac{2 b}{M^{2}}+\frac{2}{M^{3}}\right) e^{-M b} ; \\
& l_{9}=\left(S b^{2}+\frac{2}{S}\right) \cosh S b-\left(S^{2} b^{3}+2 b\right) \sinh S b ; \\
& l_{10}=\left(S b^{2}+\frac{2}{S}\right) \sinh S b-\left(S^{2} b^{3}+2 b\right) \cosh S b ; \\
& l_{11}=\sqrt{\frac{\pi}{2}} M^{3}\left(b^{4}+\frac{b^{3}}{M}+\frac{3 b^{2}}{M^{2}}+\frac{6 b}{M^{3}}+\frac{6}{M^{4}}\right) e^{-M b} ; \\
& l_{12}=\left(6-\sigma^{2} b^{2}\right) ; \\
& l_{13}=2 \sigma^{2} b^{5} ; \\
& l_{14}= \\
& \left(\left(\sigma^{2}-M^{2}\right) b^{3}-6 b\right) \sinh S b-\left(3 M b^{2}+\frac{6}{M}+M^{3} b^{4}-\frac{\sigma^{2} b^{2}}{M}-M \sigma^{2} b^{4}\right) \operatorname{coshSb} ; \\
& l_{15}= \\
& \left(\left(\sigma^{2}-M^{2}\right) b^{3}-6 b\right) \cosh S b-\left(3 M b^{2}+\frac{6}{M}+M^{3} b^{4}-\frac{\sigma^{2} b^{2}}{M}-M \sigma^{2} b^{4}\right) \sinh S b ; \\
& l_{16}=\frac{\cosh S a}{S a}-a \sinh S a ; l_{17}=\frac{\sinh S a}{S a}-a \operatorname{coshSa} ; \\
& l_{18}=\operatorname{asinh} S a-\left(\frac{1}{s}+S a^{2}\right) \operatorname{coshS} a \\
& l_{19}=\operatorname{acosh} S a-\left(\frac{1}{s}+S a^{2}\right) \sinh S a \\
& l_{20}=a^{3} .
\end{aligned}
$$

